\title{
Depresión en personas diagnosticadas con cáncer
}

Sandra Carolina Valencia Lara*
Universidad Nacional de Colombia Universidad Santo Tomás

Recibido: Marzo 30 de 2006 Revisado: Mayo 2 de 2006 Aceptado: Mayo 30 de 2006

\section{Resumen}

En el ámbito de la salud, se han observado conexiones entre la enfermedad médica y morbilidad psicológica. La condición biológica y psicológica propia de algunas enfermedades, parece favorecer la aparición de síntomas psicológicos que a menudo son confundidos con los síntomas de la enfermedad o los síntomas producidos por los tratamientos de la misma. Se considera que los trastornos psiquiátricos son frecuentes en personas con cáncer, en especial la depresión. En este artículo se hace una revisión de los factores de riesgo para este trastorno reportados en la literatura, así como la formulación del diagnóstico y tratamiento en personas con cáncer.

Palabras clave: Depresión, cáncer, factores de riesgo, diagnóstico, tratamiento.

\section{Abstract}

In the health services has been observed connections between medical illness and psychological morbidity. The biological situation in some chronic diseases produces psychological symptoms than confused with the medical

* Correspondencia: Sandra Carolina Valencia Lara, Facultad de Psicología, Universidad Santo Tomás. Cra. 9 No. 51 - 11, Bogotá, Colombia. Correo electrónico: scvalencial@gmail.com. 
condition. A proportion of cancer patients develop psychiatric disorders following the diagnosis of cancer and requires treatment, because exist premorbidly or the increased strain as a result of the disease and its treatment. This article provides an overview of the risk factor, diagnosis and screening procedures treatment modalities for depression in patients with cancer.

Key words: depression, cancer, risk factors, diagnosis, treatment.

En el siglo XX la esperanza de vida aumentó entre 30 y 40 años en promedio para la población mundial, gracias a la reducción de algunos riesgos para la salud asociados con las enfermedades infecciosas. Estos cambios han dado como resultado un fenómeno de transición demográfica en el que se pasa de sociedades tradicionalmente jóvenes a sociedades en las que crece rápidamente el número de personas de edad madura y anciana. Además, los cambios en los hábitos de consumo y estilo de vida, junto con el envejecimiento de la población, se asocian con enfermedades como el cáncer, las cardiopatías, los accidentes cerebrovasculares, la enfermedad mental y la diabetes (Organización Mundial de la Salud, 2002).

Muchas personas que reciben el diagnóstico de alguna de las enfermedades crónicas enunciadas arriba experimentan algún tipo de malestar emocional, que se considera normal, ante un evento catastrófico como el cáncer (Massie \& Popkin, 1998); la respuesta emocional frente al diagnóstico se presenta en tres fases así: una inicial, en la que las personas reaccionan con incredulidad o rechazo y desesperación; sigue una de disforia, en la que están ansiosas y presentan un estado de ánimo depresivo, anorexia, insomnio, irritabilidad, concentración deficiente y alteración de las actividades cotidianas; y por último, una de adaptación en la que los individuos se ajustan a la nueva información, confrontan aspectos que se les presentan, tienen razones para estar optimistas y reanudan sus actividades cotidianas (Massie, Spiegel, Lederberg \& Holland, 1996).
Los indicadores de una adaptación exitosa incluyen el involucramiento activo y continuo en las actividades cotidianas, la habilidad para minimizar algunas alteraciones en los roles vitales y la capacidad para regular el malestar emocional (Vallejo, Gastó, Cardoner \& Catalán, 2002; Nicholas \& Veach, 2000; Harrison \& Maguire, 1994). Los síntomas de malestar psicológico pueden desaparecer al cabo de unas semanas con el apoyo de la familia, los amigos y el equipo médico. Sin embargo, algunos pacientes continúan experimentando altos niveles de depresión y ansiedad que persisten por semanas o meses; esta persistencia no es adaptativa y con frecuencia requiere tratamiento psicológico (Massie \& Popkin, 1998).

Por esto es importante que se evalúe a la persona para identificar la presencia de un trastorno psicológico, ya que aumenta la mortalidad, la falta de adhesión al tratamiento, los costos sanitarios por hospitalizaciones prolongadas, la interacción de las medicaciones prescritas para los dos trastornos, la influencias bioquímicas de los estados de ánimo en el sistema inmune y la incapacidad laboral (Peveler, Carson \& Rodin, 2002; Parikh, Lam \& CANMAT Depression Group, 2001; Roca \& Arroyo, 1996). Sin embargo, los trastornos depresivos en pacientes con patologías médicas son poco diagnosticados o ignorados, no son tratados y rara vez se someten a tratamiento adecuado, lo que es objeto de complicaciones como el suicidio (Charlson \& Peterson, 2002; Agency for Health Care Policy and Research [AHCPR], 1997; Roca \& Arroyo, 1996). 
En este artículo se hace una revisión de la literatura sobre depresión y cáncer, en la que se muestran factores de riesgo, evaluación, diagnóstico y tratamiento de este trastorno psicológico en pacientes oncológicos.

\section{Factores de riesgo de depresión en población oncológica}

Los primeros estudios acerca de depresión mayor en cuidado primario encontraron que existía una alta prevalencia de la misma, estimada entre $4.8 \%$ y $13 \%$ (Coyne, Thompson, Klinkman \& Nease, 2002; Katon \& Ciechanowski, 2002) en poblaciones con diagnósticos médicos; estudios recientes muestran una prevalencia entre $6 \%$ y $14 \%$, un tiempo de incidencia de $15 \%$ y dos a tres veces más común en las personas hospitalizadas o con enfermedades crónicas (Fisch, 2004) que en otros grupos de personas. La interpretación de estas prevalencias es compleja, ya que los pacientes deprimidos pueden presentar síntomas que no les permiten alcanzar los criterios diagnósticos y no mostrar un deterioro sustancial, por lo que a menudo son subdiagnosticadas y subtratadas por los médicos. (Coyne et al., 2002; Katon \& Ciechanowski, 2002). La presencia de depresión mayor se asocia con el incremento de la experimentación de síntomas, reducción de la calidad de vida y pobres resultados médicos (Allen, Cull \& Sharpe, 2003; Spiegel \& Giese-Davis, 2003; Jones, 2001).

Se considera que los pacientes en cuidado primario pueden experimentar ansiedad y depresión debido a las secuelas fisiológicas de las enfermedades o sus tratamientos, respuesta psicológica ante la enfermedad, presencia de otras condiciones médicas, número total de síntomas físicos, trastornos relacionados con el abuso de sustancias, uso de múltiple fármacos, deprivación sensorial, pérdida de funcionamiento físico, aislamiento, dependencia de otros, cambios en los estilos de vida, estigma, autoadministración de tareas, amenazas a la dignidad y a la autoestima, estrés, autovaloración pobre del estado de salud, perturbaciones de las transiciones vitales y disminución de recursos (Bisshop, Kriegsman, Beekman \& Deeg, 2004; Kim, Braun \& Kunik, 2001; Jackson, O’Malley
\& Kroenke, 1998; Spiegel, 1996). La enfermedad confronta a las personas con retos y amenazas que incluyen la experimentación de dolor, desfiguramiento y cambios en las expectativas futuras (Spiegel \& Giese-Davis, 2003; Ritterband \& Spielberger, 2001).

El trastorno psiquiátrico que se asocia con más frecuencia con el cáncer es la depresión (Ritterband \& Spielberger, 2001). En la mayoría de estudios internacionales, se encuentra una rango de prevalencia de morbilidad psicológica en cáncer entre $4.5 \%$ y $50 \%$ (Raison \& Miller, 2003; Ciaramella \& Poli, 2001; Zabora, Brintzenhofeszoc, Curbow, Hooker \& Piantadosi, 2001; Breitbart, 1994; McDaniel \& Nemeroff, 1993; Derogatis, Morrow \& Fetting, 1983); autores como Ritterband \& Spielberger (2001) afirman que la prevalencia puede alcanzar hasta un $85 \%$ en los pacientes oncológicos. Las tasas de prevalencia de depresión varían y parecen afectar entre el $9 \%$ al $58 \%$ de las personas diagnosticadas con cáncer (Massie, 2004; Pirl, 2004), variabilidad debida a la falta de estandarización en términos de edad y género de la población estudiada, etapa y sitio de la enfermedad, tamaño de la muestra, instrumentos de evaluación, puntos de corte, tipo de medida y criterios diagnósticos empleados (Massie, 2004; Trask, 2004).

Las altas tasas de depresión en cáncer pueden deberse al estigma, temores, percepciones y síntomas asociados con la enfermedad. Para la mayoría de personas, el temor principal es morir de manera dolorosa; a esto se suma el temor de volverse incapaces y dependientes, tener alteraciones en su apariencia, experimentar cambios en las funciones de su cuerpo y perder la compañía de las personas allegadas. Todas estas respuestas normales ante una crisis de este tipo se asemejan a las descritas ante pérdidas (Massie \& Popkin, 1998).

Como se había mencionado antes, la adaptación psicosocial al cáncer es un proceso continuo de ajuste de la persona a una variedad de estresores vitales relacionados con la enfermedad. Los indicadores de una adaptación exitosa incluyen el involucramiento activo y continuo en las activida- 
des cotidianas, la habilidad para minimizar algunas alteraciones en los roles vitales y la capacidad de regular el malestar emocional. Una variedad de factores influye en la adaptación como los derivados del cáncer, del paciente y de la sociedad, la etapa del ciclo vital y el curso clínico del cáncer (Vallejo et al., 2002; Nicholas \& Veach, 2000; Breitbart, 1994; Harrison \& Maguire, 1994). Para efectos expositivos, se tendrá en cuenta la clasificación de Harrison \& Maguire (1994) de factores derivados del paciente, la enfermedad, la interacción paciente-enfermedad y el ambiente que influyen en el proceso de adaptación a la enfermedad oncológica.

Las características de los pacientes se refieren a variables sociodemográficas como edad, género, estado civil y clase social. A pesar de la evidencia de que los trastornos de ansiedad y depresión son muy prevalentes en la población general en el rango de mediana edad, los resultados de pacientes oncológicos son contradictorios. En efecto, algunos estudios no muestran relación entre edad y morbilidad psicológica, pero, en los que se observa dicho efecto, los pacientes mayores parecen encontrarse mejor (Valente \& Saunders, 1997; Mor, Allen \& Malin, 1994; Given, Given \& Stommel, 1994; Noyes, Kathol \& Debelius, 1990). Estos resultados podrían explicarse porque estos pacientes experimentan menos malestar existencial y pocas dificultades, en comparación con los jóvenes, quienes, al enfrentarse ante una eventualidad médica, pueden encontrar más dificultades en el afrontamiento de las limitaciones prácticas derivadas de la enfermedad y el tratamiento (Valente \& Saunders, 1997; Given et al., 1994; Noyes et al., 1990).

Respecto a las tasas de morbilidad psicológica, teniendo en cuenta el género, se encuentran contradicciones en los estudios: en algunos estudios las tasas son iguales para hombres y mujeres, y, en otros, son más altas entre las mujeres. Si se comparan áreas específicas de ajuste se puede observar que las mujeres experimentan más dificultades con respecto a la imagen corporal y problemas emocionales, en contraposición con los hombres que informan dificultades prácticas con los sínto- mas físicos o el deterioro en su funcionamiento. Las limitaciones de estos estudios se deben principalmente a la inclusión de pacientes cuyos diagnósticos son dependientes del género como el cáncer de mama o de testículo, por lo que se requiere investigación con grupos donde esta variable se mezcle (Noyes et al., 1990; Derogatis, et al., 1983).

Si se analiza el estado civil, los estudios en pacientes con cáncer muestran poca relación entre éste y morbilidad psicológica, en contraposición a la evidencia suministrada por estudios con población general, en los cuales los síntomas depresivos son más comunes entre divorciados o separados, que en los solteros o los casados. La posible explicación del fenómeno en el escenario oncológico radica en que probablemente es más importante la calidad de las relaciones interpersonales que el estado civil (Stefanek, Derogatis \& Shaw, 1987; Derogatis et al., 1983). También son de anotar los resultados contradictorios de los estudios con pacientes oncológicos en cuanto a la relación entre clase social y morbilidad psicológica (Derogatis et al.,1983). En el análisis de Nicholas \& Veach (2000), se encontró que el estrato socioeconómico se relaciona con la incidencia, estilos de afrontamiento, recurrencia, sobrevida y tasas de mortalidad en los pacientes con cáncer.

Dentro de los factores del paciente, también deben considerarse factores psicológicos previos como personalidad, autoestima e historia psicológica (Nordin, Berglund, Glimelius \& Sjöden, 2001; Nicholas \& Veach, 2000; Noyes et al., 1998). Junto a lo anterior, se incluyen los tratamientos psiquiátricos preexistentes y los trastornos mentales comórbidos, el abuso de sustancias, las experiencias previas de enfermedad y las pérdidas significativas recientes. Los pocos estudios con pacientes oncológicos que analizan la relación entre personalidad (neuroticismo) y depresión, muestran resultados similares en cuanto a niveles altos de neuroticismo y posterior desarrollo de depresión (Nicholas \& Veach, 2000; Noyes, Holt \& Massie, 1998). Por otro lado, los escasos estudios que evalúan el papel de la autoestima como predictor de ajuste psicológico han encontrado que la autoestima alta se relaciona con niveles bajos de 
síntomas depresivos y niveles altos de bienestar (Schoroevers, Ranchor \& Sanderman, 2003). Con respecto a la historia psicológica previa, se ha encontrado que las personas que sufrieron depresión o ansiedad en el pasado, tuvieron una mayor probabilidad de volver a experimentarlas ante un estresor mayor como el cáncer (Nicholas \& Veach, 2000; Noyes et al., 1998).

Los factores asociados a la enfermedad y el tratamiento se refieren a las variables que describen la enfermedad, tratamiento y procesos relacionados, resultado de los estresores situacionales (2000). Se considera que las personas con cáncer son vulnerables a la depresión porque su organismo experimenta alteraciones metabólicas y endocrinas a raíz de la enfermedad y el tratamiento que es debilitante, lo que modifica las respuestas inmune y de dolor (Ballenger et al., 2001).

Existe una fuerte relación entre estado físico y morbilidad psicológica, ya que los niveles altos de esta se observan en los pacientes más enfermos, en los que tienen puntajes bajos en ejecución y en los que presentan síntomas no placenteros persistente tales como dolor (Ciaramella \& Poli, 2001; Nordin et al., 2001; Nicholas \& Veach, 2000; Valente \& Saunders, 1997). En la fase terminal de la enfermedad, por ejemplo, los pacientes muestran múltiples síntomas físicos y psicológicos que les causan sufrimiento a ellos y sus familias, y que dificultan el diagnóstico de un trastorno psicológico (Block, 2000, 2001; Donelly \& Walsh, 1995).

Los estudios que han intentado evidenciar morbilidad psicológica por grupo de enfermedad muestran hallazgos contradictorios, ya que muchos han fracasado en su intento de vincularla con el tipo de cáncer (Ciaramella \& Poli, 2001; Nicholas \& Veach, 2000;) y otros reportan tipos de cáncer altamente asociados como el orofaríngeo, pancreático, mama y pulmón (Massie, 2004). En el estudio de Ciaramella \& Poli (2001), la presencia de metástasis fue un factor que condicionó la presencia de un episodio depresivo mayor, probablemente debido a que el diagnóstico de depresión aumenta con el avance de la enfermedad.
Dentro del tratamiento, la cirugía se asocia con impacto en imagen corporal, aislamiento, problemas psicosexuales y dificultades en el funcionamiento social (Hutton \& Williams, 2001; Nicholas \& Veach, 2000). Por esto, el temor y el duelo son respuestas probables ya que la cirugía y otros tratamientos para el cáncer son drásticos y pueden provocar reacciones de pérdida (Maguire \& Murray, 1998). Los pacientes con medicación psicotrópica por algún diagnóstico psiquiátrico previo, son vulnerables a la exacerbación los síntomas asociados al trastorno diagnosticado (esquizofrenia, depresión mayor, trastornos de ansiedad, enfermedad maníaco-depresiva), por lo que el psiquiatra evita suspender la medicación en el período de tiempo cercano a la cirugía. Después de la cirugía se presenta depresión cuando los resultados de la misma son desfavorables, por lo que el período postoperatorio es de recuperación del procedimiento y de confrontación y adaptación a la pérdida y a la posible muerte. Además, se pueden presentar reacciones emocionales relacionadas con el sitio de la cirugía (lengua, laringe, cara, mamas, genitales, colon) y la pérdida de funcionalidad (Jacobsen, Roth \& Holland, 1998).

La quimioterapia, terapia hormonal, inmunoterapia y la radioterapia se consideran como procedimientos invasivos que perturban la vida de los pacientes y les recuerda constantemente que tienen cáncer (Knobf, Pasacreta, Valentine \& McCorkle, 1998). Junto a esto, la inmunoterapia, basada en el uso de citokinas, y la quimioterapia pueden inducir síntomas depresivos en los pacientes que están sometidos a estos tratamientos (Raison \& Miller, 2003). Además, en estos tratamientos es usual que las personas tengan una serie de síntomas que causan malestar tales como fatiga, náuseas, vómitos y dolor (Nicholas \& Veach, 2000; Dimeo, Stieglitz, Novelli-Fischer, Fetscher, \& Keul, 1999;). Estos síntomas pueden abarcar desde los moderados hasta los severos en cuanto al funcionamiento cotidiano del paciente, imagen corporal, relaciones familiares, relaciones sexuales y malestar psicológico general (Greenberg, 1998; Jacobsen et al., 1998; Knobf et al., 1998). 
Por las razones expuestas arriba, es importante evaluar si las respuestas psicológicas del paciente se relacionan con el efecto secundario de alguna droga o a cambios en el sistema nervioso central En cuanto a los efectos neuropsiquiátricos secundarios de las drogas anti-cáncer, se puede afirmar que los síntomas como delirio, confusión, concentración deficiente, depresión, alucinaciones, desorientación, somnolencia, irritabilidad, agitación, deterioro de la memoria y alteraciones de la cognición son el resultado de disfunciones cerebrales y cerebelosas, de la toxicidad en el sistema nervioso central o incluso de encefalopatías. Al terminar el tratamiento es posible que los síntomas puedan explicarse mejor por la carencia de enfermedad mensurable, menos contacto con los proveedores de cuidado de salud y la persistencia de síntomas que pueden manifestarse en ansiedad, depresión, tristeza, malestar emocional general, aumento del temor de recurrencia e incertidumbre (Knobf et al., 1998).

Entre las variables de la enfermedad y el tratamiento también es importante analizar el problema de la discapacidad. Este término se define como alguna restricción o carencia de habilidad para realizar alguna actividad de la manera o dentro del rango que se consideró normal para un ser humano. Los pacientes oncológicos pueden experimentar discapacidades en diversas ocasiones en el curso clínico de la enfermedad. Las limitaciones para la adaptación psicosocial pueden ser el resultado de diversos tipos de alteraciones funcionales causadas por la enfermedad en sí misma, por los tratamientos del cáncer y/o por sus efectos secundarios. También pueden ser el resultado de la fatiga, la anorexia o caquexia, linfedema, deficiencias neuropsicológicas y otros síntomas relacionados. Algunos síntomas pueden ser agudos pero de corta duración como la náusea y la alopecia, otros pueden ser crónicos como las disfunciones sexuales o los problemas de memoria, y otros de duración variable como la fatiga. Esos síntomas pueden afectar la habilidad del paciente para permanecer activo e involucrarse en la vida diaria, y en algún momento del curso clínico pueden deteriorar la adaptación psicosocial (Kelly, Ghazi \& Caldwell, 2002;
Bruce, 2002; Berard, 2001; Nicholas \& Veach, 2000; Williamson, 2000).

En la investigación, se ha encontrado una interacción entre el paciente y los recursos con los que cuenta para afrontar la enfermedad y ajustarse a la misma (Nicholas \& Veach, 2000). Este es el concepto de afrontamiento, compuesto por los estilos y estrategias: los primeros se refieren a las características disposicionales, estables y duraderas (rasgo), que un individuo muestra en diferentes situaciones; las segundas se refieren a una gran variedad de respuestas cognitivas, conductuales e instrumentales (estado) diseñadas para resolver problemas específicos y así aliviar estresores específicos, mediante los cuales se intenta modificar la situación y disminuir la percepción de amenaza (centrada en el problema) o regular la respuesta emocional provocada por dicha amenaza (centrada en la emoción) (Rowland, 1989; Lazarus y Folkman, 1984).

Numerosos estudios sugieren que el estilo de afrontamiento puede contribuir a la etiología o resultado físico del cáncer. Sin embargo, no hay acuerdo en cuanto al papel del afrontamiento en los pacientes oncológicos. Confrontación, redefinición del problema y cumplimiento con la asesoría médica son las respuestas que los estudios asocian con una mejor adaptación psicológica. Por el contrario, las respuestas con menor efectividad son las que incluyen intentar olvidar el problema, la aceptación estoica, la reducción de la tensión mediante el cigarrillo y el alcohol, la distracción, el retiro social y la culpabilización de sí mismo y de los demás. Sin embargo, también se demuestra que, en las fases agudas del diagnóstico y de la enfermedad, la negación o distracción pueden disminuir la ansiedad. A largo plazo, la tendencia a controlar emociones, ver el futuro sin esperanza, verse a sí mismo incapaz de realizar cambios que le permitan manejar la situación y adoptar la aproximación de indefensión o fatalismo puede incrementar el riego de morbilidad psicológica (Hutton \& Williams, 2001; Nicholas \& Veach, 2000).

Los factores del ambiente se refieren a las estructuras sociales relacionadas con la persona y de las 
cuales puede recibir soporte social. Los efectos positivos del soporte social se explican de dos maneras: incremento en emociones, cogniciones y conductas positivas y amortiguamiento indirecto de los estresores vitales asociados con el cáncer. El soporte social debe analizarse de dos formas: el soporte estructural o la red social de personas con quienes el paciente tiene contacto regular y las funciones del soporte que incluyen las dimensiones emocional, informacional y la asistencia instrumental específica (Nicholas \& Veach, 2000).

El apoyo social inadecuado es un factor de riesgo para la morbilidad psicológica en una variedad de ambientes. Los aspectos del soporte social que son de particular importancia para los pacientes oncológicos son tres: primero, las incertidumbres y temores experimentados por los pacientes oncológicos son el resultado probable de una elevada necesidad de soporte emocional, de la oportunidad de discutir sentimientos y recibir información; segundo, la naturaleza aterradora y estigmatizante de la enfermedad les dificulta la obtención de apoyo adecuado a los que experimentan problemas; y tercero, el tipo de apoyo que necesita la persona es diferente porque la enfermedad, y el tratamiento, es un proceso dinámico. Parece ser que el soporte social en pacientes oncológicos no tiene un impacto sobre el funcionamiento social, pero influye en la percepción de bienestar (Nordin et al., 2001; Noyes et al., 1998). Respecto de las demandas propias del cáncer y de sus tratamientos, debe agregarse que el malestar psicológico puede deteriorar el apoyo social al desgastarlo si se convierte en una crisis crónica, y que las personas que brindan apoyo pueden responder disminuyendo su ayuda (Bolger, Foster, Vinokur \& Ngi, 1996)

Por último, debe considerarse el contexto vital y dentro de éste, los esquemas relacionados con la salud y la etapa del desarrollo. Los esquemas se refieren a las representaciones sociales de la enfermedad que tiene el paciente en contraposición al conocimiento del médico, las cuales pueden diferir en cuanto a la consecución de objetivos. La etapa de desarrollo se refiere a la fase del ciclo vital en la que se encuentra la persona y que se relaciona con los objetivos y las tareas biológicas, personales y sociales (Nicholas \& Veach, 2000).

\section{Diagnóstico de depresión en personas con cáncer}

La investigación muestra que la depresión es comórbida a otros trastornos mentales (trastornos de ansiedad, obsesivo-compulsivos, de personalidad, psicóticos, etc.) y biológicos (cáncer, hipotiroidismo, etc.), haciendo difícil identificarla en el cuidado primario (Charlson \& Peterson, 2002; AHCPR, 1997; Roca \& Arroyo, 1996). Roca y Arroyo (1996) enuncian como factores que se deben tener en cuenta para realizar un diagnóstico diferencial del trastorno depresivo cuando hay una patología médica; pérdida de tono, anhedonia, tristeza, despertar de madrugada y mejoría vespertina del ánimo, conductas psicomotoras enlentecidas, antecedentes familiares de depresión, mejoría de síntomas depresivos como respuesta al ejercicio físico, incapacidad para experimentar placer ante el contacto psicosocial, fatiga, anorexia, pérdida de peso y dolor.

El desarrollo de estos trastornos en cuidado primario parece depender de varios factores como la patología médica específica (curso, evolución y pronóstico, que condicionan la cronicidad), la existencia de historia psiquiátrica previa, carencia de apoyo social y las estrategias de afrontamiento utilizadas para enfrentar la enfermedad (Vallejo et al., 2002; Roca \& Arroyo, 1996).

Dadas las características de los trastornos del estado de ánimo en cuidado primario, es aconsejable realizar una entrevista que permita dilucidar la presencia de signos y síntomas que permitan efectuar un diagnóstico diferencial. Este instrumento de evaluación permite obtener información acerca del uso de sustancias o medicaciones que puedan causar la sintomatología depresiva, determinar la existencia de otro trastorno no afectivo que pueda asociarse con el estado de ánimo y descartar causas alternativas; además de la entrevista, se recomienda practicar una valoración médica con el 
fin de detectar la existencia de patologías que biológicamente puedan causar o estar asociadas con la depresión (AHCPR, 1997). Sin embargo, el uso de entrevistas diagnósticas en escenario primario tiene limitaciones como la dificultad de evaluar a todos los posibles pacientes en riesgo de trastorno afectivo y el alto costo de su implementación.

Por esta razón, con el fin de reducir costos y el tiempo que generaría la realización de entrevistas diagnósticas, se propone la realización de procedimientos psicológicos de tamizaje (Palmer \& Coyne, 2003; Coyne, Thompson \& Racioppo, 2001). Este tipo de procedimiento permite una evaluación temprana de personas en riesgo de presentar un trastorno y que pueden requerir de intervención psicológica prioritaria (Valencia, 2005; Zabora, 1998), ya que la falta de diagnóstico y tratamiento de la depresión generan a los pacientes sufrimiento, deterioro personal y costos sociales (Palmer \& Coyne, 2003; Coyne et al., 2001).

Entre las ventajas de usar instrumentos de tamizaje se encuentran la facilidad y rapidez de administración y calificación (Sharp \& Lipsky, 2002; Endicott, 1984); además, estos instrumentos pueden ser administrados por profesionales diferentes a los psicólogos (médicos, enfermeras, por ejemplo), diligenciados por las mismas personas, utilizados con poblaciones de riesgo (personas con enfermedades crónicas, dolor, ambientes familiares estresantes, fases postnatales, vejez) y reproducidos fácilmente (Ebell, 2004; Sharp \& Lipsky, 2002; Massie \& Popkin, 1998). Esta última ventaja permite una fácil evaluación del estado mental de la persona en el tiempo, teniendo en cuenta valoraciones iniciales y de seguimiento. La limitación más importante es que valora sólo algunos aspectos cognitivos que se consideran centrales en el trastorno psicológico (Massie \& Popkin, 1998).

Para elegir una medida de tamizaje se deben tener en cuenta varios aspectos: primero, la extensión del instrumento, es decir, la pertinencia de elegir herramientas largas o cortas; segundo, las características de la población a ser tamizada; tercero, las propiedades psicométricas de los instrumen- tos; cuarto, el tiempo requerido para completar la medida y para calificar el instrumento; quinto, la facilidad de uso; y, sexto, el costo de obtener la medida. Otra consideración importante en el momento de la elección es el formato de los instrumentos, ya que los pacientes pueden reportar sus síntomas en pruebas de lápiz y papel o al contestar un par de preguntas simples en una entrevista personal o telefónica (Valencia, 2005; Pirl, 2004; Williams, Pignone, Ramírez \& Perez, 2002; LloydWilliams, 2001). Sin embargo, alrededor de este último aspecto, se discute si un instrumento compuesto por un par de preguntas simples sobre el trastorno es válido y eficiente para identificar a las personas que requieren una valoración más profunda (Williams et al., 2002; Coyne et al., 2001).

El procedimiento de tamizaje como tal se conoce como de valoración en dos etapas, en el que primero se evalúa una población blanco y luego se realiza una entrevista con el fin de realizar una estimación exacta del trastorno y su historia (Ebell, 2004; Sharma, Avasthi, Chakrabarty \& Varma, 2002; Sharp \& Lipsky, 2002; Coyne et al., 2001; LloydWilliams, 2001). Si el instrumento proporciona un puntaje, se determina si el que obtuvo la persona es superior al de corte establecido estadísticamente y en el que se supone los síntomas del trastorno se consideran significativos; si es así, se realiza una entrevista estructurada para valorar la persona según los criterios diagnósticos asociados al trastorno, establecidos en sistemas de clasificación tradicionales como el DSM y el CIE (Sharp \& Lipsky, 2002; Williams et al., 2002; Lloyd-Williams, 2001; Hasselblad \& Hedges, 1995). La necesidad de la entrevista estructurada radica en que las medidas de tamizaje no permiten determinar características diagnósticas como duración de los síntomas, grado de deterioro y trastornos psiquiátricos comórbidos.

Para realizar un procedimiento de tamizaje se recomienda obtener información de varias fuentes, considerar la condición médica de las personas porque en algunos casos se asocia con altas tasas de trastornos psicológicos, determinar el nivel de deterioro cognitivo y la existencia de deficiencias sensoriales que puedan presentar algunas pobla- 
ciones y considerar la posibilidad de la inflación de las tasas de casos identificados en personas con deterioros cognitivos (Sharp \& Lipsky, 2002; Coyne et al., 2001; Parker, Hilton, Hadzi-Pavlovic \& Bains, 2001).

En el momento en que se ha determinado que una persona con cáncer se clasifica en riesgo de un trastorno depresivo, el clínico debe realizar una entrevista confirmatoria teniendo en cuenta criterios diagnósticos. Entre los utilizados de manera más frecuente en personas con cáncer se encuentran Clasificación Internacional de Enfermedades (ICD), el sistema de Criterios Diagnósticos de Investigación (RCD), el Criterio Endicott y el Manual Estadístico y Diagnóstico de la Asociación Americana de Psicología (DSM-IV).

El DSM-IV define la depresión como la presencia de ánimo deprimido o la pérdida del interés o placer en todas las actividades por un período de al menos dos semanas; para realizar el diagnóstico de este trastorno, la persona debe presentar 4 síntomas cognitivos y somáticos adicionales, los cuales deben producirle deterioro o malestar significativo. Para este trabajo son importantes dos tipos o subcategorías de depresión: trastorno depresivo mayor (MDD) y trastorno distímico/distimia. El trastorno depresivo mayor se caracteriza por uno o más períodos depresivos, la ausencia de episodios maniacos y está definido por un ánimo deprimido o anhedonia (disminución del interés o placer en las actividades cotidianas), acompañado por un número de síntomas asociados. Un número específico de esos síntomas debe estar presente diariamente, aproximadamente por 2 semanas, representar un cambio en el funcionamiento previo y malestar o deterioro en las áreas social, ocupacional y otras importantes. En el trastorno distímico los síntomas no son lo suficientemente severos para alcanzar el criterio de trastorno depresivo mayor (Trask, 2004; Sellick \& Crooks, 1999; American Psychiatric Association, 1994).

Además de los síntomas mencionados, para la formulación del diagnóstico, las personas deben presentar otros síntomas como cambio en el peso o apetito (ganancia o pérdida), alteraciones en el sueño (insomnio o hipersomnio), agitación o enlentecimiento psicomotor observable, fatiga, culpa inapropiada, pobre concentración y pensamientos recurrentes de muerte o de suicidio (Newport \& Nemeroff, 1998). Dada las características de la enfermedad oncológica, también debe considerarse la formulación de otros diagnósticos como trastorno de ajuste con ánimo deprimido o mixto o duelo. Sin embargo, las limitaciones intrínsecas de algunos sistemas de clasificación pueden permitir que muchos casos subclínicos se pierdan. Muchos individuos que tienen síntomas de depresión, no alcanzan los criterios de trastorno depresivo mayor o aún trastorno distímico (Sellick \& Crooks, 1999). Además, muchos médicos pueden pasar por alto los síntomas psicológicos ya que esperan que los pacientes los reporten espontáneamente; asumen que son una reacción normal al diagnóstico y esperan que las personas conozcan por qué los experimentan (Valente \& Saunders, 1997).

Es evidente en los reportes de diferentes investigaciones la variabilidad en el diagnóstico de un trastorno depresivo en las personas con cáncer debido a la utilización de diferentes criterios diagnósticos, muestras de pacientes con diversos cánceres, tipos de instrumentos y puntos de corte utilizados, y a la similitud de los síntomas producidos por la enfermedad y su tratamiento y los síntomas depresivos (Trask, 2004; Raison \& Miller, 2003; Newport \& Nemeroff, 1998). Para evaluar de la manera más exacta posible el trastorno depresivo en las personas con cáncer, Cavenaugh (1995) describe cuáles son los componentes de la formulación de un diagnóstico válido:

- Validez aparente: es el grado en el que una agrupación particular de síntomas describe un trastorno.

- Validez descriptiva: es el grado para el cual los síntomas de un trastorno particular son únicos a ese trastorno y así permiten distinguirlo de otros.

- Validez predictiva: los síntomas de un diagnóstico particular sirven para pronosticar el curso de la enfermedad y su tratamiento. 
- Validez de constructo: es el grado en que la teoría racional puede construirse para explicar la etiología y patofisiología del trastorno.

Para efectos clínicos prácticos, Cohen-Cole, Brown \& MCDaniel (1993) proponen cuatro aproximaciones conceptuales en la realización de un diagnóstico de depresión en las personas que tienen una enfermedad física:

- Aproximación inclusiva: incluye todos los síntomas de depresión mayor, aun si ellos pueden ser secundarios a la enfermedad médica. Este método es de sensibilidad alta, especificidad baja y no se centra en la etiología.

- Aproximación exclusiva: elimina de la lista de los criterios diagnósticos, síntomas secundarios a la enfermedad como fatiga y anorexia.

- Aproximación sustitutiva: sugiere sustituir los síntomas cognitivos como autocompasión, cavilación, temporadas de llanto o pesimismo por síntomas neurovegetativos tales como alteraciones en el apetito, en el sueño y fatiga.

- Aproximación etiológica: propone que cada síntoma debe ser tomado en cuenta solamente si no es secundario a la enfermedad médica.

Finalmente, además de la depresión en personas con enfermedades médicas como el cáncer, también debe considerarse el suicidio. Los factores que ubican al paciente con cáncer en un alto riesgo de suicidio son pobre pronóstico y enfermedad avanzada, depresión y desesperanza, dolor incontrolado, delirio, historia psicológica previa, historia de intentos previos de suicidio o historia familiar de suicido, historia de muerte reciente de amigos o esposo(a), historia de abuso de alcohol, y poco apoyo social. Otros factores de riesgo incluyen sexo masculino, edad avanzada, presencia de fatiga, cáncer oral, faríngeo, de pulmón, gastrointestinal, urogenital o de mama (Druss \& Pincus, 2000; Massie \& Popkin, 1998; Sutor et. al., 1998; Breitbart, 1994).

\section{Tratamiento de la depresión en personas con cáncer}

Los tratamientos para la depresión pueden dividirse en biológicos y psicológicos. Los tratamientos biológicos que se usan de manera preferencial son la medicación y la terapia electroconvulsiva. Las medicaciones antidepresivas se pueden clasificar en tres clases: antidepresivos tricíclicos, inhibidores selectivos de la recaptación de la serotonina e inhibidores de las monoaminoxidasas. El tratamiento de la depresión con esta medicación es efectivo en casos de depresión cuya severidad vaya de moderada a severa; el conjunto de síntomas que predicen una respuesta favorable a los antidepresivos son disminución del apetito, insomnio, ataque agudo, historia familiar de depresión y una historia personal de respuesta a los antidepresivos o terapia electroconvulsiva (Angelino \& Treisman, 2001; DeRubeis, Young \& Dalhsgaard, 1998).

Los antidepresivos tricíclicos han mostrado ser eficaces en el tratamiento de la depresión. Como todas las medicinas, tienen efectos secundarios, tales como taquicardia, hipotensión postural y sedación. El uso de estos medicamentos con pacientes suicidas conlleva serios riegos debido a la toxicidad neurológica y cardiovascular.

El uso de inhibidores selectivos de la recaptación de la serotonina están indicados para los mismos casos en los que se utilizan los antidepresivos tricíclicos. Se prefiere para tratar la depresión debido a su efectividad comparable a los tricíclicos pero con efectos secundarios reducidos. Los efectos secundarios comunes de los inhibidores selectivos de la recaptación de la serotonina son gastrointestinales y sexuales incluyendo naúsea, diarrea y disfunción sexual. También se han reportado disminución del apetito, nerviosismo y temblores (DeRubeis et al., 1998).

Los inhibidores de las monoaminooxidasas son indicados en general en los casos de depresión atípica que no responde adecuadamente a los tricíclicos y 
a los inhibidores selectivos de la recaptación de la serotonina. La categoría de depresión atípica incluye depresión no melancólica, depresión con ansiedad, sensibilidad al rechazo, dolor crónico o síntomas vegetativos invertidos como aumento del apetito e hipersomnio. Mientras se supone que los tricíclicos y lo inhibidores de la recaptación de la serotonina alteran el funcionamiento de la neurotransmisión al inhibir la recaptación de aminas dentro de la hendidura presináptica, los inhibidores de las monoaminooxidasas actúan a través de la inhibición de la monoaminooxidasa o enzima que provoca una degradación o pérdida de la efectividad de los neurotransmisores cateco-lamina y serotonina. Entre los efectos secundarios de los inhibidores de las monoaminooxidasas se encuentran jaquecas, boca seca, insomnio, constipación, sedación, visión borrosa y nausea. Son de interés particular las interacciones potenciales no placenteras y aún fatales entre estos inhibidores con alimentos que contienen altos niveles de tiramina. Al ingerir inhibidores de las monoaminooxidasas, se deben evitar quesos añejados, vinos tintos, salsa de soya, pescado ahumado y otros alimentos que contengan la sustancia ya referida (DeRubeis et al., 1998).

El tratamiento farmacológico de los síntomas severos de depresión en cáncer se indica en personas que cumplen los criterios de episodios depresivos mayores, ya que se ha comprobado su eficacia en estos casos (Breitbart, 1994). Se debe considerar la toxicidad y seguridad de los medicamentos antidepresivos, dado que los tratamientos para el cáncer (quimioterapia, radioterapia, inmunoterapia) pueden debilitar al paciente y dejarlo en estado vulnerable. Un perfil de seguridad no favorable para antidepresivos tricíclicos puede evitar su uso en personas con cáncer (Berard, 2001).

La terapia electroconvulsiva se considera generalmente como un último recurso de tratamiento, utilizado en personas que requieren hospitalización por la severidad de la depresión o que no responden adecuadamente a medicaciones antidepresivas. Esta terapia es considerada primaria cuando se necesita una respuesta rápida al trata- miento, cuando el riesgo de la medicación es grande en comparación con la terapia electroconvulsiva, cuando hay una historia de respuesta pobre a la medicación o historia de respuesta positiva a la terapia electroconvulsiva o cuando la persona prefiere este tratamiento a otros. Ha mostrado especial efectividad cuando la depresión está acompañada por psicosis o retardo psicomotor marcado y estupor depresivo. Aunque el mecanismo a través del cual la terapia electroconvulsiva alcanza los efectos antidepresivos no se conoce muy bien, se sabe que la inducción de un ataque de gran mal es esencial para su éxito; una posible explicación es que la liberación de norepinefrina y dopamina durante el ataque lleve a un mejoramiento general del funcionamiento del sistema de aminas (DeRubeis et al., 1998).

Los tratamientos psicológicos para la depresión que se utiliza generalmente la terapia cognitiva, la terapia cognitivo-conductual y conductual. La terapia cognitiva de Beck para la depresión asume que las personas deprimidas frecuentemente mantienen un pensamiento negativo que al ponerse al escrutinio será encontrado erróneo. Los terapeutas en terapia cognitiva enseñan a los pacientes a atender sus pensamientos, algunos de los cuales son distorsionados. Luego, enseñan a las personas formas efectivas que les permitan reconsiderar sus pensamientos, mediante preguntas que permitan verificar la validez de dichos pensamientos (DeRubeis et al., 1998).

La terapia cognitiva también ha sido llamada cognitivo-conductual porque se enfoca también en acciones. En las etapas tempranas del tratamiento, se emplean técnicas conductuales para oponerse al fracaso de la persona deprimida para iniciar una acción que puede ser placentera o tener un sentido de logro. El terapeuta ayuda a la persona a descomponer la tarea en pequeños componentes y comprometerse en actividades que evita. El objetivo del terapeuta es el compromiso de los pacientes con el mundo y el descubrimiento de creencias distorsionadas. La terapia cognitiva está basada en habilidades, y se espera que la persona no solo se beneficie de la intervención terapéutica sino 
que lo que aprendió lo aplique a un amplio rango de situaciones y actividades (DeRubeis et al., 1998).

La terapia conductual se centra en la adquisición de habilidades, especialmente sociales, y en el enriquecimiento del ambiente mediante la identificación, planeación y compromiso en la realización de actividades potencialmente reforzantes. Entre las aproximaciones conductuales al tratamiento de la depresión tenemos terapia de solución de problemas, que se basa en el presupuesto de que las personas deprimidas han perdido (o nunca han tenido) las habilidades necesarias para resolver los problemas vitales (DeRubeis et al., 1998).

La decisión de tratar a un paciente por depresión en el escenario de cuidado primario, mediante una terapia psicológica, no se toma siempre sobre la base de criterios diagnósticos rígidos, sino sobre el juicio de severidad y duración de los síntomas que experimenta la persona y la probabilidad de una recuperación espontánea en un ambiente de apoyo (Fisch, 2004). Los objetivos del tratamiento psicológico en cáncer son reducir el dolor emocional y mejorar el ánimo, la capacidad de afrontamiento, la autoestima, el sentido de control y la solución de problemas. A menudo, los pacientes con cáncer son remitidos en momentos de crisis de la enfermedad como recurrencia, comienzo de un nuevo tratamiento, fracaso del tratamiento actual o cuando los pacientes se perciben a ellos mismos como terminales. La referencia es a menudo una emergencia y los pacientes en crisis aceptan de buena gana una intervención; un modelo de intervención útil es la combinación de aspectos educacionales y técnicas cognitivas, junto con el suministro de apoyo emocional y social brindado por la familia, amigos, grupo religioso y "pacientes veteranos” (Newport \& Nemeroff, 1998).

Entre los tratamientos disponibles para estos pacientes se encuentran la psicoterapia individual, psicoterapia de grupo, hipnoterapia, psicoeducación multimedia, entrenamiento de relajación y retroalimentación, grupos de autoayuda, terapia cognitiva, terapia de apoyo, entrenamiento en solución de problemas y en habilidades sociales (Hopko, Armento, Hunt, Bell \& Lejuez, 2005;
Newport \& Nemeroff, 1998; Breitbart, 1994). Varios estudios han evaluado la eficacia de las intervenciones de soporte psicosocial en pacientes deprimidos, encontrándose que no solamente son más efectivos en el afrontamiento sino que incrementan el tiempo de sobrevida. Los estudios indican que una intervención psicosocial adecuada no solamente incrementa la calidad de vida del paciente con cáncer, sino también la extienden (Newport \& Nemeroff, 1998).

La última opción de tratamiento de la depresión es la combinación de la farmacoterapia con la psicoterapia. Las razones que se argumentan para hacerlo son los potenciales beneficios evidenciados en aumento de la respuesta al tratamiento, mejoramiento de la calidad de vida, reducción de las tasas de recaída/recurrencia y facilitación de dosis bajas de medicación junto con el aumento de la adherencia al tratamiento. La combinación de los tratamientos puede hacerse de tres maneras: concurrente (psicoterapia más farmacoterapia), secuencial (adicionar otro tratamiento para quienes no responden o responden parcialmente al tratamiento en la fase aguda de tratamiento) y mezclado (cambiar a psicoterapia para la fase de mantenimiento del tratamiento después de la respuesta a la farmacoterapia en la fase aguda) (Berard, 2001; Segal, Kennedy, Cohen \& CANMAT Depression Group, 2001). Como ya se indicó, el manejo óptimo de los síntomas depresivos en personas con cáncer se realiza cuando se combina la psicoterapia de apoyo, las técnicas cognitivo-conductuales y la medicación antidepresiva (Breitbart, 1994).

\section{Referencias}

Agency for Health Care Policy and Research. (1997). Detection and Diagnosis. Depression in Primary Care. Clinical Practice Guideline 1.

Allen, K., Cull, A. \& Sharpe, M. (2003). Diagnosing major depression in medical outpatients: acceptability of telephone interviews. Journal of Psychosomatic Research, 55, 385387. 
American Psychiatric Association. (1994). diagnostic and statistical manual of mental disorders ( $4^{\mathrm{a}}$. ed.) Washington, DC: Autor.

Angelino, A. F. \& Treisman, G. J. (2001) Major depression and demoralization in Cancer patients: diagnostic and treatment considerations. Support Care Cancer, 9, 344349.

Ballenger, J. C., Davidson, T. R. T., Lecrubier, Y., Nutt, D. J., Jones, R. D. \& Berard, R. M. F. (2001). Consensus Statement on Depression, Anxiety, and Oncology. J. Clin Psychiatry, 62 (Suppl 8), 64-67.

Berard, R. M.F. (2001) Depression and anxiety in oncology: the psychiatrist's perspective. $J$ Clin Psychiatry, 62(suppl 8), 58-61.

Bisshop, M. A., Kriegsman, D. M. W., Beekman, A. T. F. \& Deeg, D. J. H. (2004). Chronic diseases and depression: the modifying role of psychosocial resources. Social Science \& Medicine, 59, 721-723.

Block, S. (2000). Assessing and managing depression in the terminally ill patient. Annals Of Internal Medicine, 132, 209-218.

Block, S. (2001). Psychological considerations, growth, and trascendence at the end of life: the art of the possible. JAMA, 285, 289872905.

Bolger, N., Foster, M., Vinokur, A. D. \& Ngi, R. (1996). Close Relationships and adjustment to a Life Crisis: The Case of Breast Cancer. Journal of Personality and Social Psycholog, 10, 283-294.

Breitbart, W. (1994). Psycho-oncology: Depression, Anxiety, Delirium. Seminars in Oncology, 21(6), 754-769.

Bruce, M. L. (2002). Psychosocial Risk actors for Depressive Disorders in Late Life. Biol. Psychiatry, 52, 175-184.
Cavenaugh, S. (1995). Depression in the medically ill: critical issues in diagnostic assessment. Psychosomatics, 36, 58-59.

Charlson, M. \& Peterson, J. C. (2002). Medical Comorbidity and Late Life Depression: What is known and What are the Unmet Needs?. Biol. Psychiatry, 52, 226-235.

Ciaramella, A. \& Poli, P. (2001). Assessment of depression among cancer patients: the role of pain, cancer, type and treatment. Psychooncology, 10, 156-165.

Cohen-Cole, S. A, Brown, F. W. \& McDaniel, S. (1993). Diagnostic assessment of depression in the medically ill. En: Stoudemire, A. \& Fogel, B. (Eds.). Principles of medical psychiatric (pp 53-68). New York: Oxford University Press.

Coyne, J. C., Thompson, R., Klinkman, M. S. \& Nease Jr., D. E. (2002). Emotional disorders in primary care. Journal of Consulting and Clinical Psychology, 70, 798-809.

Coyne, J. C., Thompson, R. \& Racioppo, M. W. (2001). Validity and Efficiency of Screening for history of depression by Self-Report. Psychological Assessment, 13, 163- 170.

Derogatis, L. R., Morrow, G. R. \& Fetting, J. (1983). The prevalence of Psychiatric disorders among cancer patients. Journal of American Medical Association, 249, 751 757.

DeRubeis, R. J., Young, P. R. \& Dahlsgaard, K. K. (1998). Affective disorders. En: Bellack, A. S. y Hersen, M. (Eds.). Comprehensive Clinical Psychology. Pp 339-366. Versión electrónica.

Dimeo, F. C., Stieglitz, R-D., Novelli-Fischer, U., Fetscher, S. \& Keul, J. (1999). Effects of physical activity on the fatigue and psychologic status of cancer patients during chemotherapy. Cancer, 85, 2273-2277. 
Donelly, S. \& Walsh, D. (1995). The symptoms of advanced cancer. Seminars in Oncology, 22,67-72.

Druss, B. \& Pincus, H. (2000). Suicidal ideation and suicide attempts in general medical illness. Archives of Internal Medicine, 160, 15221526.

Ebell, M. H. (2004). Routine screening for depression, alcohol problems, and domestic violence. American Family Physician, 69(10), 2421-2422.

Endicott, J. (1984). Measurement of Depression in Patients With Cancer. Cancer, 53 (10Suppl.), 2243-2249.

Fisch, M. (2004). Treatment of depression in cancer. Journal of the National Cancer Institute Monographs, 32, 105-111.

Given, C. W., Given, B. A. \& Stommel, M. (1994). The impact of age, treatment, and symptoms on the physical and mental health of cancer patients. Cancer, 74 (suppl.), 2128-2138.

Greenberg, D. B. (1998). Radiotherapy. En: Holland, J (Ed.). Psycho-oncology (pp. 269-277). New York: Oxford University Press.

Harrison, J. \& Maguire, P. (1994). Predictors of Psychiatry morbidity of cancer patients. British Journal of Psychiatry 165, 593-598.

Hasselblad, V. \& Hedges, L. V. (1995). Meta-analysis of screening and diagnostic test. Psychological Bulletin, 117, 167-178.

Hopko, D. R., Armento, M. E. A., Hunt, M. K., Bell , J. L. \& Lejuez, C. W. (2005). Behavior Therapy for depressed cancer patient in primary care. Psychotherapy: Theory, Research, Practice, Training, 42, 236-243.

Hutton, J. M. \& Williams, M. (2001). An investigation of psychological distress in patients who have been treated for head and neck cancer. British Journal of Oral and Maxillofacial Surgery, 39, 333-339.

Jackson, J. L., O’ Malley, P. G. \& Kroenke, K. (1998). Clinical predictor of mental disorders among medical outpatients: validation of de "S4" model. Pyschosomatics, 39, 431-436.

Jacobsen, P. B., Roth, A. J. \& Holland, J. (1998). Surgery. En: Holland, J. Psycho-oncology (pp. 257-268). New York: Oxford University Press.

Jones, R. D. (2001). Depression and anxiety in oncology: the oncologist's perspective. $J$ Clin Psychiatry, 62(suppl 8), 52-55.

Katon, W. \& Ciechanowski, P. (2002). Impact of major depression on chronic medical illness. Journal of Psychosomatic Research, 53, 859863.

Kelly, C., Ghazi, F. \& Caldwell, K. (2002). Psychological distress of cancer and clinical trial participation: a review of literature. European Journal of Cancer Care, 11, 6-15.

Kim, F. S., Braun, U. \& Kunik, M. E. (2001). Anxiety and depression in medically older adults. Journal of Clinical Geropsychology, 7, 117130.

Knobf, M. T., Pasacreta, J. V., Valentine, A. \& McCorkle. (1998). Chemotherapy,

Hormonal Therapy, and Inmunotherapy. En: Holland, J (Ed.). Psycho-oncology (pp. 277288). New York: Oxford University Press.

Lazarus, R. S. \& Folkman, S. (1984). Stress, appraisal, and coping. New York: Springer.

Lloyd-Williams, M. (2001). Screening for depression in palliative care patients: Areview. European Journal of Cancer Care, 10, 31-35.

Maguire, P. \& Murray P. C. (1998). Surgery and loss of body parts. BMJ, 316, 1086-1088. 
Massie, M. J. (2004). Prevalence of depression in patients with cancer. Journal of the National Cancer Institute Monographs, 32, 57-71.

Massie, J. M. \& Popkin, M. K. (1998). Depressive disorders. En: J. (Ed.). Psycho-oncology (Pp. 518-540). New York: Oxford University Press.

Massie, J. M. Spiegel, L., Lederberg, M. \& Holland, J. C. (1996). Complicaciones psiquiátricas de los pacientes con cáncer. En: Murphy, G. P., Lawrence Jr. W. y Lenhard, R.E. (Eds.). Oncología Clínica. Manual de la American Cancer Society. Washington:Organización Panamericana de la salud y la Oficina Regional de la Organización Mundial de la Salud. pp. 769-783.

McDaniel, J. S. \& Nemeroff, C. B. (1993). Depression in the cancer patient, diagnostic, biological, and treatment aspects. En: Chapman, C. R. \& Fole, K. M. (Eds.). Current and Emerging Issues in Cancer Pain: Research and Practice (pp. 1-19). New York: Raven Press.

Mor, V., Allen, S. \& Malin, M. (1994). The psychosocial impact of cancer on older Versus younger patients and their families. Cancer, 74, 2118-2127.

Newport, D. J. \& Nemeroff, C. B. (1998). Assessment and treatment of depression in the cancer patient. Journal of Psychosomatic Research, 45, 215-237.

Nicholas, D. R. \& Veach, T. A. (2000). The Psychosocial assessment of the adult Cancer patient. Professional Psychology: Research and Practice, 31, 206-215.

Nordin, K., Berglund, G., Glimelius, B. \& Sjöden, P.-O. (2001). Predicting anxiety and depression among cancer patients: a clinical model. European Journal of Cancer, 37, 376384.

Noyes Jr, R., Holt, C. S. \& Massie, M. J. (1998). Anxiety disorders. En: Holland, J. C. Ed.).
Psycho-oncology (pp 548-563). New York: Oxford University Press.

Noyes, R., Kathol, R. G. \& Debelius E. L. (1990). Distress associated with cancer as measured by the illness scale. Psychosomatics, 31, 321330.

Organización Mundial de la Salud (2002). Informe sobre la salud en el mundo 2002: Reducir los riesgos y promover una vida sana. Ginebra: Autor.

Palmer, S. C. \& Coyne, J. C. (2003). Screening for depression in medical care: pitfalls, alternatives, and revised priorities. Journal of Psychosomatic Research, 54, 279-287.

Parikh, S. V., Lam, R. W. \& CANMAT Depresión Work Group. (2001). Clinical Guidelines for the Treatment of Depressive Disorders: Definitions, Prevalence, and Health Burden. The Canadian Journal of Psychiatry 46(Suppl 1), 13S-20S.

Parker, G., Hilton, T., Hadzi-Pavlovic, D. \& Bains, J. (2001). Screening for depression in the medically ill: the suggested utility of a cognitive-based approach. Australian and New Zealand Journal of Psychiatry, 35, 474480.

Peveler, R., Carson, A. \& Rodin, G. (2002). Depression in medical patients. BMJ, 325, 149-152.

Pirl, W. F. (2004). Evidence report on the occurrence, assessment and treatment of depression in cancer patients. Journal of the National Cancer Institute Monographs, 32, 32-39.

Raison, C. L. \& Miller, A. H. (2003). Depression in cancer: new developments regarding diagnosis and treatment. Biol. Psychiatry, 54, 283-294.

Ritterband, L. M. \& Spielberger, C. D. (2001). Depression in a cancer population. Journal 
of Clinical Psychology in Medical Settings, 8, 85-93.

Roca B. M. \& Arroyo, M. B. (1996). Depresión y enfermedad médica. En: Roca B, M. \& Arroyo, M. B (Eds.). Trastornos depresivos en patología médicas. Barcelona: Masson.

Rowland, J. H. (1989). Interpersonal resources: Social support. En: Holland, J.C. Rowland, J. H. (Eds.). Handbook pf Psycho-oncology: Psychological care of the Patient with cancer ( $\mathrm{pp}$ 58-71). New York: Oxford University Press.

Segal, Z. D., Kennedy, S. H., Cohen, N. L. \& CANMAT Depression Group. (2001). Clinical guidelines for the treatment of depressive disorders: combinig psychoterapy and pharmacotherapy. The Canadian Journal of Psychiatry, 46(Suppl 1), 59S-62S.

Sellick, S. M. \& Crooks, D. N. (1999). Depression an cancer: an appraisal of the literature for prevalence, detection, and practice guideline development for psychological interventions. Psycho-oncology, 8, 315-333.

Sharma , P., Avasthi, A., Chakrabarti, S., \& Varma, S. (2002). Depression among hospitalized medically ill patients: a two-stage screening study. Journal of Affective Disorders, 70, 205- 209.

Sharp, L. K. \& Lipsky, M. S. (2002). Screening for depresión across the lifespan: A Review of measures for use in primary care settings. American Family Physician, 66(6), 10011008.

Shoroevers, M. J., Ranchor, A. V. \& Sanderman, R. (2003). The role of social support and self esteem in the presence and course of depressive symptoms: a comparisons of cancer patients and individuals from the general population. Social Science \& Medicine, 57, 375-385.
Spiegel, D. (1996). Cancer and depression. British Journal of psychiatry, 168(suppl.30), 109116.

Spiegel, D. \& Giese-Davis, J. (2003). Depression and cancer: mechanisms and disease progression. Biol. Psychiatry, 54, 269-282.

Stefanek, M. E., Derogatis, L. R. \& Shaw, A. (1987). Psychological distress among oncology outpatients: prevalence and severity as measured with Brief Symptom Inventory. Psychosomatics, 28, 530-579.

Sutor, B., Rummans, T., Jowey, S., Krahn, L., Martin, M., O'Connor, M. K., et al. (1998). Major depression in Medically III Patients. Mayo Clinics Proceedings. 73(4), 329-337.

Trask, P. C. (2004). Assessment of depression in cancer patient. Journal of the National Cancer Institute Monographs 32, 80-92.

Valencia L. S. C. (2005). El procedimiento de tamizaje en psicología clínica y de la salud.Avances en Medición, 3, 173-176.

Valente, S. M.. \& Saunders, J. N. (1997). Diagnosis and treatment of major depression among people cancer. Cancer Nursing, 20, 168-177.

Vallejo, R. J. Gastó, F. N:, Cardoner A., N. \& Catalán C., R. (2002). Comorbilidad de los trastornos afectivos. Barcelona: Ars Medica.

Williams Jr., J. W., Pignone, M., Ramirez, G. \& Perez S., C. (2002). Identifying depression in primary care: a literature synthesis of case-finding instruments. General Hospital Psychiatry, 24, 225-237.

Williamson, G. M. (2000). Extending the Activity Retristicion Model of Depressed Affect:Evidence From a Sample of Breast Cancer Patients. Health Psychology, 19, 339347. 
Zabora, J., Brintznhofeszoc, K., Curbow, B., Hooker, C. \& Piantadosi, S. (2001). The prevalence of psychological distres by cancer site. Psycho-oncology, 10, 19-28.
Zabora, J. R. (1998). Screening procedures for psychosocial distress. En: Holland, J. (Ed.). Psycho-oncology (pp 653-675). New York: Oxford University Press. 